Oliveira, V.M.; Brasil, M.R.; Mattes, V.V.; Álvarez, V.A.E. y Souza, J. (2021). The Reception of Norbert Elias' Work in Brazilian Physical Education. Revista Internacional de Medicina y Ciencias de la Actividad Física y el Deporte vol. 21 (82) pp. 337-353 Http://cdeporte.rediris.es/revista/revista82/artrecepcion1248.htm

DOI: https://doi.org/10.15366/rimcafd2021.82.009

\title{
LA RECEPCIÓN DEL TRABAJO DE NORBERT ELÍAS EN LA EDUCACIÓN FÍSICA BRASILEÑA
}

\section{THE RECEPTION OF NORBERT ELIAS' WORK IN BRAZILIAN PHYSICAL EDUCATION}

\author{
Oliveira, V.M. ${ }^{1}$; Brasil, M.R. ${ }^{1}$; Mattes, V.V. ${ }^{1}$ Álvarez, V.A.E. ${ }^{2}$ y Souza, J. ${ }^{3}$ \\ 1 Estudiante de doctorado del Programa de Postgrado en Educación Física de la Universidad \\ Estatal de Maringá (Brasil) oliveira vm@hotmail.com, brasillmr@hotmail.com.br. \\ vero edf@hotmail.com \\ ${ }^{2}$ Estudiante de graduación de educación física y salud de la Universidad Católica del Maule \\ (Chile) vicente.espindola@alu.ucm.cl \\ 3 Profesor del Programa de Postgrado en Educación Física de la Universidad Estatal de \\ Maringá (Brasil) y coordinador del Observatorio de Educación Física y Deporte (OEFE-UEM- \\ Brasil) julianoedf@yahoo.com.br
}

\section{Agradecimientos}

Los investigadores agradecen a la Coordinación de Perfeccionamiento del Personal de Nivel Superior (CAPES) por el apoyo financiero a los investigadores.

Código UNESCO I UNESCO Code: 630199 Otras Especialidades Sociológicas (Sociología del deporte) / Other Sociological Specialties (Sociology of Sport)

Clasificación Consejo de Europa / Council of Europe Classification: 16 Sociología del deporte / Sociology of Sport

Recibido 28 de mayo de 2019 Received May 28, 2019

Aceptado 7 de diciembre de 2019 Accepted December 7, 2019

\section{RESUMEN}

Este estudio tiene como objetivo investigar el proceso de recepción y apropiación del trabajo de Norbert Elías en el campo de la Educación Física brasileña. Para cumplir tal objetivo, se realizó un análisis de las ocho principales revistas científicas socioculturales del país. Después de la recolección de datos, se encontraron 452 estudios que tenían alguna relación con la referencia del autor. Entre los principales resultados, se señala que el proceso de recepción del trabajo del sociólogo comenzó alrededor de la década de 1990, intensificándose solo en la presente década. Aunque ha habido un crecimiento de estudios en relación con las contribuciones de Elías en la literatura, al analizar los textos de muestra, se dio cuenta que solo el $17,61 \%$ tenía una apropiación profunda del modus operandi elíasiano. Además, 
la mayoría de los estudios estaban relacionados con el deporte y el ocio, lo que indica la posibilidad de que el autor sea explorado en otros contextos.

PALABRAS CLAVE: Educación Física; Norbert Elías; producción científica; Sociología del Deporte

\section{ABSTRACT}

This study aims to investigate the process of reception and appropriation of Norbert Elias' work in the Brazilian Physical Education field. To achieve this objective, an analysis was made of the eight main scientific journals of the socio-cultural area in the country. After data collection, 452 studies were found that had some relationship with the author's reference. Among the main results, it is noted that the process of receiving the work of the sociologist began around the 1990s, being intensified only in the present decade. Although there has been a growth of studies in connection with Elias' contributions in the literature, when analyzing the sample texts, it was found that only $17.61 \%$ had a rigorous appropriation of the eliasian way of working. In addition, most studies were linked to sports and leisure, indicating the possibility of the author being explored in other contexts.

KEYWORDS: Norbert Elias; Physical Education; Scientific production; Sociology of Sport.

\section{INTRODUCIÓN}

Norbert Elías fue uno de los grandes intelectuales de la escena sociológica del siglo XX (Dunning y Hughes, 2013). No obstante, a diferencia de otros autores, el sociólogo alemán enfrentó dificultades de consolidación en el campo académico, siendo él mismo, en sus términos, un outsider frente al establecimiento sociológico de la época (Elías, 2000, 2001a). En líneas generales, por colocarse en una posición de contrariedad a la sociología clásica, sobre todo en lo que se refiere a la tensión "individuo" versus "sociedad", Elías tuvo su trabajo por algún tiempo silenciado (Mennell, 2006). Sin embargo, no por la calidad de su proyecto creador, sino porque el sociólogo presentaba una teoría general de la sociedad que caminaba en la dirección diametralmente opuesta al pensamiento sociológico vigente a principios del siglo XX.

De esta forma, al ponerse en oposición a la sociología tradicional y anunciarse como un innovador en el campo, su trabajo se reconoció más sistemáticamente sólo alrededor de 1970, siendo esta recepción, en un primer momento, más acentuada en los países en que el sociólogo vivió, a saber, Alemania, Francia, Inglaterra, los cuales, incluso, sirvieron de laboratorio empírico para la composición de su obra magna, "El proceso de la civilización" (Górnicka Liston y Mennell 2015, Oliveira, 2018). En esa tesitura, si el proceso de recepción se dio de forma tardía en el contexto internacional, era relativamente previsible algún retraso en la divulgación de la obra de Elías en países de América Latina, como fue el caso de Brasil. 
En ese sentido, en tierras brasileñas, los primeros movimientos más incisivos acerca de la circulación del trabajo de Elías se reportan a mediados de los años 1990 y fueron estimulados, en gran medida, por agentes del campo de la Educación Física (EF), en particular, por algunos investigadores de la Universidad Estatal de Campinas (UNICAMP), responsables por una serie de iniciativas de difusión del referencial elíasiano en el país, a ejemplo de la consolidación de una red relacional con los colaboradores de una primera generación intelectual que trabajaron directamente con Norbert Elías, o sea, Johan Goudsblom, Eric Dunning, Stephen Mennell, Cas Wouters, entre otros (Gebara, 2014).

Es importante reconocer que esta proximidad de los investigadores de EF con la sociología elíasiana no surgió al azar. Por el contrario, esa relación es explicada también en virtud del trabajo que el autor desarrolló en el campo de la Sociología del Deporte y del Ocio. En síntesis, de los años 1960 hasta próximo a su muerte en 1990, Elías destinó en su agenda de investigación, un gran espacio al estudio del deporte y del ocio, legando una teoría general que posibilitaba una comprensión sofisticada y rigurosa de esos objetos tan importantes al campo de la EF (Souza, Starepravo y Marchi-Júnior, 2014). Esto, a su vez, es uno de los elementos decisivos que influyó en la recepción del trabajo del autor en el área (Oliveira, 2018), sobre todo por la necesidad de ampliación de los posibles horizontes de entendimiento de tales fenómenos, mas allá de las dimensiones aisladas, es decir, biológicas, culturales, psicológicas, políticas y así sucesivamente.

En este panorama, con el paso de los años, las contribuciones del autor comenzaron a volverse cada vez más visibles en estudios socializados en el campo de la EF, especialmente en las principales revistas de divulgación científica relacionadas con el área (Pedraz, 2010; Souza, Starepravo y MarchiJúnior, 2014; Alfrey y Gard, 2017; Righeto y Reis, 2017; Black y Fielding-Lloyd, 2017; Manrique Arribas, 2018; Lavega-Burgués y Navarro-Adelantado, 2019; Morales-Rosillo; Petro y Bonilla, 2019). No obstante, aunque se verificó este uso del trabajo de Elías en las investigaciones del espacio académico en análisis, no está claro en la literatura cómo ha ocurrido esta recepción. De esta forma, identificada esa laguna en la literatura, en especial en lo que concierne a la lectura de los casi 30 años de inmersión del referencial teórico elíasiano en Brasil, el presente estudio tuvo como objetivo verificar el proceso de recepción y apropiación del trabajo de Norbert Elías en algunas de las más relevantes revistas científicas de la EF brasileña.

\section{DISEÑO METODOLÓGICO DE LA INVESTIGACIÓN}

Para suplir la investigación propuesta en este estudio, seleccionamos como fuente de datos algunas de las principales revistas científicas del área de EF en Brasil. Entre los criterios de elección de las revistas, optamos por: [1] revistas que contemplaban la recepción de estudios socioculturales; [2] que presentaban estratificaciones Capes entre cualidades A hasta B2 (o sea, revistas bien calificadas de acuerdo con los criterios de evaluación científica en Brasil); [3] que tenían libre acceso a todos los números publicados, así como 
publicación trimestral o cuatrimestral. Una vez delimitado ese perfil de muestra, llegamos a la elección de ocho revistas, a saber: Journal of Physical Education (JPE/UEM), Revista Brasileira de Ciências do Esporte (RBCE), Revista Brasileira de Educação Física e Esporte (RBEFE), Revista Licere (RL), Revista Pensar a Prática (RPP), Revista Motrivivência (RMT), Revista Motriz (RMZ) e Revista Movimento (RM).

Después de la selección de la muestra, accedemos a la plataforma virtual de cada revista y realizamos la descarga de todos los textos disponibles que comprendían la primera edición hasta el último volumen disponible. En la secuencia, con la posesión de los documentos y por medio del comando control find, definimos como descriptores de búsqueda la palabra clave "Norbert Elías". Para los textos digitalizados, la búsqueda fue realizada manualmente para capturar los estudios que tenían afinidad con el descriptor. Cabe destacar, que la investigación contempla hasta el último número de 2018 de cada revista, con excepción de la RBEFE que se encuentra con las actividades paralizadas desde 2017.

Posteriormente al análisis de los documentos, localizamos el total de 452 textos que poseían alguna alusión a Elías, siendo 426 artículos, 15 resúmenes, 10 reseñas y 1 entrevista. La revista que presentó el mayor número de estudios fue la RM con el total de 114 textos $(25,22 \%)$ seguidos, respectivamente, por la RBCE 74 (16,37\%), RL 70 (15,49\%), RMT 44 (RM) (9,74\%), RPP 40 (8,85\%), RMZ 40 (8,85\%), RBEFE 35 (7,74\%) y JPE/UEM 35 (7,74\%). Con la producción mapeada, se realizó la tabulación de las informaciones y los datos sometidos al análisis estadístico por el software Statistical Package for Social Science SPSS v. 23. La incursión numérica orbitó en torno a la estadística descriptiva, en particular, por análisis de frecuencia absoluta y relativa.

En líneas generales, a lo largo del estudio, verificamos el proceso de recepción de los trabajos que citan Elías, los agentes y las instituciones que más movilizan y parecen tener una mayor aproximación con el trabajo del sociólogo, las obras más citadas del referencial elíasiano, la apropiación teórica de los estudios y, por último, las principales temáticas en que la teoría de Norbert Elías ha sido movilizada. Tales informaciones son presentadas por medio de incursiones cuantitativas en relación con la interpretación cualitativa de los datos.

Para la verificación del tipo de apropiación (análisis cualitativo de contenido) realizada en los documentos analizados, recurrimos a la clasificación sugerida por Catani, Catani y Pereira (2001). En esta tipología, los investigadores sugieren la existencia de tres formas de apropiación de los referenciales teóricos: [1] la "incidental" que consiste en una utilización más básica, calificada por citas rápidas o apenas alusiones al autor en el cuerpo del texto (el autor referenciado generalmente aparece en notas a pie o sólo citado en las referencias); [2] la "conceptual tópica", caracterizada por un uso consciente, aunque no sistemático (el investigador moviliza algún concepto o citación del referencial para reforzar sus argumentos); y [3] el "modo de trabajo" que apunta a una movilización sistemática del referencial teórico (en ese tipo de 
apropiación, el investigador cita más obras, emplea más conceptos, en suma, hace la teoría funcionar).

\section{PANORAMA ACERCA DE LA RECEPCIÓN Y APROPIACIÓN DEL TRABAJO DE NORBERT ELÍAS EN REVISTAS CIENTÍFICAS DE LA EDUCACIÓN FÍSICA BRASILEÑA}

El primer resultado que traemos a la exposición se refiere al proceso de recepción cronológica de estudios que presentan alguna citación en el cuerpo del texto en referencia al trabajo de Elías. Para este tipo de análisis, se consideraron los 452 textos. Los datos se expresan en frecuencias absolutas en el gráfico siguiente.

Figura 1 - Número de documentos con mención a Elías en las revistas científicas de EF en escala cronológica

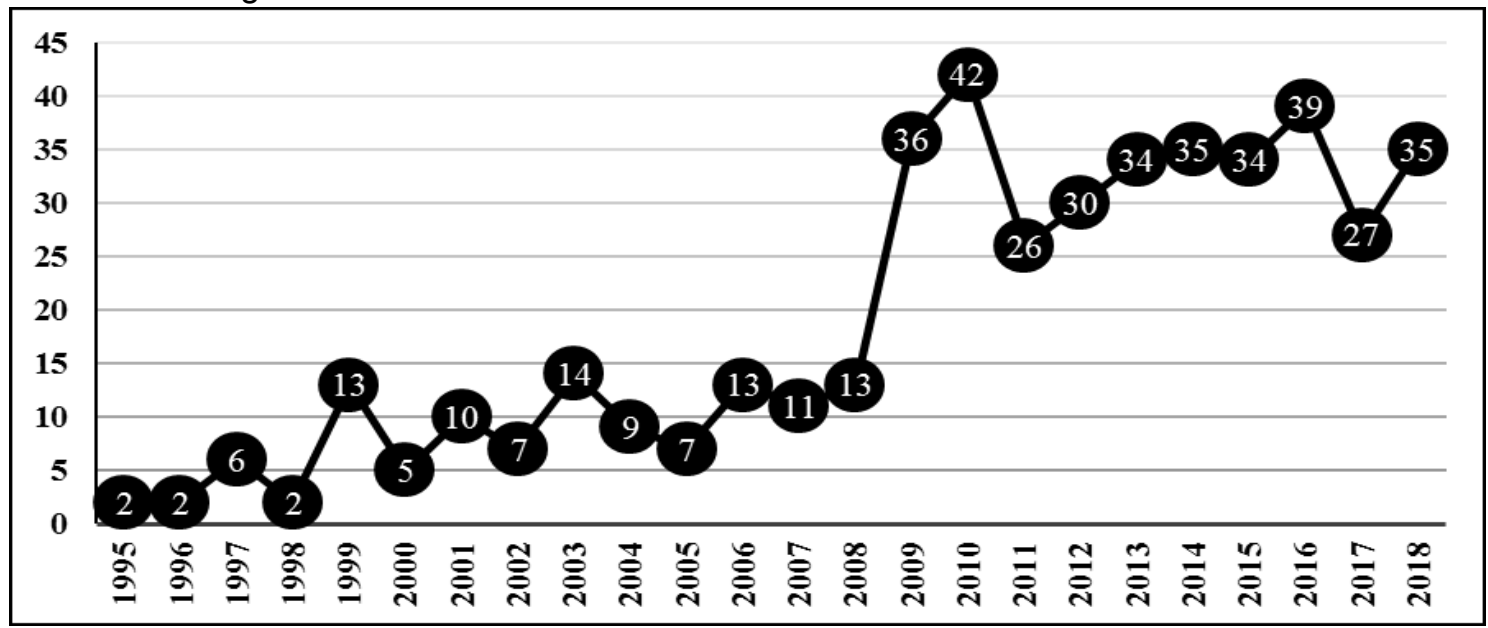

En general, es posible ver que la recepción de Elías en las principales revistas científicas del área de EF en Brasil comenzó alrededor de 1995. En ese año son encontrados dos textos, un en la RMZ y otro en la RM. La primera referencia a Elías en la $\mathrm{RMZ}$ se evidencia en una reseña crítica desarrollada por Mauro Betti (1995) con relación al libro "De la cultura del cuerpo" de Jocimar Daolio. En el transcurso del texto, Betti cita a Elías haciendo mención a la obra "El proceso de la civilización". El texto presente en la RM, se trata de un artículo sobre la temática deporte y cuerpo, titulado "¿'Jóvenes viejos' deportistas eternamente?" De Antonio Jorge Gonçalves Soares (1995). En el texto, el investigador moviliza ampliamente el referencial elíasiano, en particular, las obras "El proceso de la civilización", "La soledad de los moribundos" y "Sociología fundamental".

En 1996, se publicaron otros dos textos, uno en la RBCE y otro en la RBEFE. La primera ocurrencia se refiere a un texto también de Betti (1996), titulado "La televisión y la guerra de Pacaembu: 'populares' versus 'ciudadanos"'. En este artículo, el investigador hace usos teóricos de los dos volúmenes de la obra "El proceso de la civilización" y de dos capítulos de la colección "Deporte y ocio en el proceso de la civilización". El otro texto de 1996, en la RBEFE, fue propuesto por Helder Guerra Resende y Antonio Jorge Gonçalves Soares (1996) con el 
título "Conocimiento y especificidad de la Educación Física Escolar, en la perspectiva de la cultura corporal". Para la elaboración de este texto, los autores también se valieron del libro "Deporte y ocio en el proceso de la civilización".

Con respecto a los datos arriba citados es interesante notar que entre 1995 y 1996 ya había investigadores movilizando el referencial teórico de Elías antes de iniciar el proceso más sistemático de recepción del trabajo del autor, que tuvo lugar en gran medida con la creación del Simposio Internacional Procesos Civilizadores (SIPICs) (Gebara, 2011, 2014). Por lo tanto, se ve que a principios de 1990 existían investigadores en contacto con el marco, probablemente estimulados por las primeras publicaciones del autor en Brasil, en especial, las traducciones hechas a los portugueses por el editorial Jorge Zahar (Souza, Starepravo y Marchi-Junior, 2014).

Durante la década de 1990 y principios de la década 2000 en paralelo con el aumento de iniciativas de divulgación del trabajo de Norbert Elías, el número de investigaciones que movilizaron la teoría elíasiana continuó creciendo. Sin embargo, fue sólo cerca del final de la primera década de los años 2000, que los estudios que utilizan o citan la referencia del sociólogo se intensificaron en las revistas científicas del área investigada. Evidentemente, también hubo un aumento del número mismo de ediciones anuales de las revistas, pero aun así eso no inviabiliza el argumento de que la circulación de textos que citan o efectivamente operan a partir del referencial elíasiano vienen ganando espacio en el campo de la EF.

Discutido, en otros términos, se puede decir que, a lo largo de la última década, el referencial teórico de Elías pasó a ofrecer soporte para muchas investigaciones desarrolladas en la subárea sociocultural de EF, alcanzando varios objetos y temáticas del campo. Sin embargo, a pesar del hecho de que el número de estudios que citan a Elías en las revistas de EF revisadas aumento, eso no quiere decir que todos los artículos comparten un mismo uso de las contribuciones del autor. Por el contrario, si analizado los textos a partir de la tipología ofrecida por Catani, Catani y Pereira (2001), es posible identificar que la apropiación teórica llevada a rigor en cada artículo es diferente, conforme se puede visualizar en la siguiente figura. 
Figura 2 - Apropiación teórica del referencial elíasiano en los artículos analizados

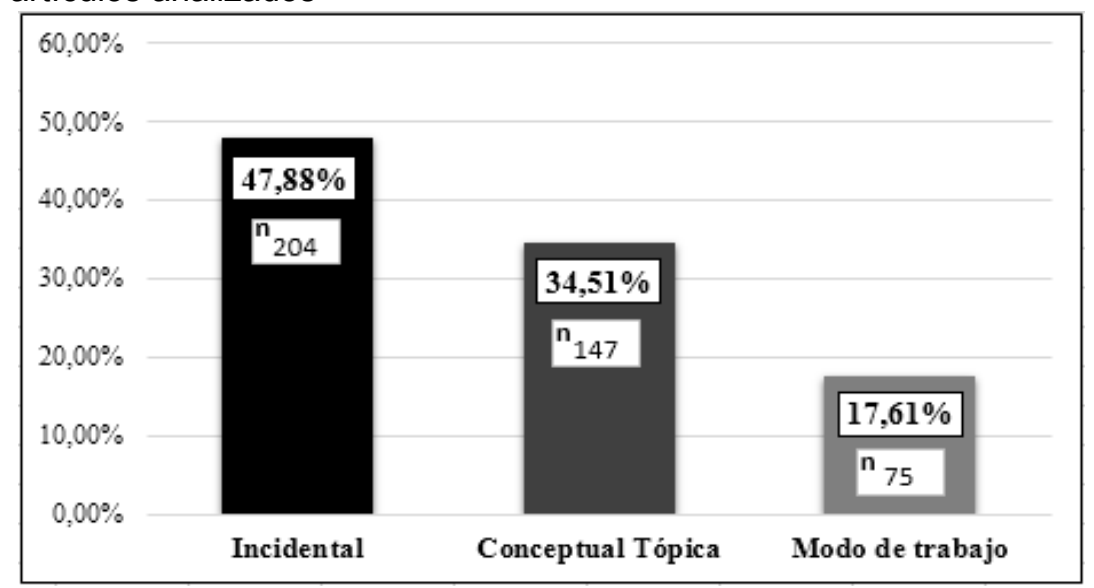

En primer lugar, es oportuno mencionar que, para los análisis de apropiación, solo se consideraron los textos en formato de artículo. Esta decisión metodológica fue tomada, porque esos documentos suelen ser más densos y necesitan una mayor inversión teórica en comparación con resúmenes o reseñas (Brasil, 2018). En ese sentido, como es observado en la figura 2, de los 426 artículos examinados, aproximadamente el $48 \%$ hacía alusión a Elías de forma "incidental". Esto es, en esos textos, la teoría de Elías fue poco citada y cuando había mención al trabajo del autor, era de manera más aligerada y con poca o sin ninguna conexión a los preceptos más generales de su "modo de trabajo".

Con un uso más equilibrado y con una movilización más heterodoxa, se visualizaron 147 artículos que satisfacían las condiciones de la apropiación entendida como "conceptual tópica". En estos estudios se verificó una mayor comunicación con el referencial, aunque claramente distanciados de una utilización más sistemática. En general, estos datos sugieren dificultades de apropiación o incluso un eclecticismo teórico que interfiere en estas producciones, al final los investigadores citan diversos autores, muchas veces, sin justificación, de forma extrapolada y sin compromisos con determinada orientación teórica.

Lógicamente, que no es posible generalizar esos análisis, teniendo en vista la existencia de situaciones en que la apropiación puede ser explicada a partir de otras variables. Por cierto, al examinar los tipos de apropiación a partir de la distribución de los artículos en cada una de las revistas investigadas, se notan algunas relaciones interesantes. 
Rev.int.med.cienc.act.fís.deporte - vol. 21 - número 82 - ISSN: 1577-0354

Tabla 1 - Número de artículos y tipo de apropiación teórica en las revistas investigadas

\begin{tabular}{cc|ccc}
\hline \multirow{2}{*}{ Revistas } & Artículos & \multicolumn{3}{|c}{ Tipo de Apropiación } \\
\cline { 3 - 5 } & 111 & $47(42,3 \%)$ & $45(40,5 \%)$ & $19(17,1 \%)$ \\
Incidental & $\begin{array}{c}\text { Conceptual } \\
\text { Tópica }\end{array}$ & $\begin{array}{c}\text { Modo de } \\
\text { trabajo }\end{array}$ \\
\hline RM & 73 & $39(53,4 \%)$ & $22(30,1 \%)$ & $12(16,4 \%)$ \\
RBCE & 67 & $29(43,3 \%)$ & $19(28,4 \%)$ & $19(28,4 \%)$ \\
RL & 42 & $26(61,9 \%)$ & $10(23,8 \%)$ & $6(14,3 \%)$ \\
RMT & 37 & $17(45,9 \%)$ & $15(40,5 \%)$ & $5(13,5 \%)$ \\
RPP & 35 & $13(37,1 \%)$ & $17(48,6 \%)$ & $5(14,3 \%)$ \\
RBEFE & 34 & $18(52,9 \%)$ & $12(35,3 \%)$ & $4(11,8 \%)$ \\
JPE (UEM) & 27 & $15(55,6 \%)$ & $7(25,9 \%)$ & $5(18,5 \%)$ \\
RMZ & & & &
\end{tabular}

En la tabla 1, vemos que la revista que más posee artículos con relación al descriptor Norbert Elías es la RM, seguido de la RBCE, dos importantes revistas en el escenario académico de la EF brasileña. En síntesis, estas revistas poseen gran "peso funcional" en la estructura del campo académico (Bourdieu, 1968), ejerciendo fuerzas que acaban, de alguna forma, atrayendo más agentes a publicar en esas revistas, en especial en la RM que actualmente reúne condiciones (Qualis A, JCR) que le permiten, a grueso modo, estar en el polo dominante si pensamos en la estructura del campo presentada por Bourdieu (1990). Así cuanto mayor el "peso funcional" de la revista, mayor el poder ejercido en el interior de las relaciones de interdependencia académica (Elías, 2010). Quizás esta sea una de las razones por las que se encontraron más artículos asociados a Elías en la RM y RBCE.

En paralelo a las dos revistas mencionadas, es necesario reconocer la importancia de la RL en el proceso de recepción de los estudios elíasianos. Aunque esta revista posea menos artículos que la RM y la RBCE, la RL es la que tiene mayor porcentaje de artículos con apropiación del "modo de trabajo" del autor alemán. Esto sugiere, que los investigadores del ocio han tratado de dar rigor analítico a sus esfuerzos, posiblemente apoyados por el uso del trabajo "Deporte y ocio en el proceso de la civilización" (Elías y Dunning, 1992) en que el sociólogo proporciona una teoría general para la comprensión de ese fenómeno que constituye el marco editorial de la RL.

Aun explorando el proceso de recepción y apropiación del trabajo elíasiano en el campo de la EF brasileña, también fue posible descubrir quiénes son los investigadores que más publicaron textos relacionados al referencial teórico en cuestión: 
Rev.int.med.cienc.act.fís.deporte - vol. 21 - número 82 - ISSN: 1577-0354

Tabla 2 - Investigadores que más citan a Elías en las revistas científicas de EF

\begin{tabular}{cc|ccc}
\hline \multirow{2}{*}{ Investigador } & \multirow{2}{*}{ Artículos } & \multicolumn{3}{|c}{ Tipo de Apropiación } \\
\cline { 3 - 5 } & & Incidental & $\begin{array}{c}\text { Conceptual } \\
\text { Tópica }\end{array}$ & $\begin{array}{c}\text { Modo de } \\
\text { trabajo }\end{array}$ \\
\hline MARCHI JUNIOR, W & 32 & $10(31,3 \%)$ & $7(21,9 \%)$ & $15(46,9 \%)$ \\
SOUZA, J. & 18 & $5(27,8 \%)$ & $4(22,2 \%)$ & $9(50,0 \%)$ \\
STAREPRAVO, F.A. & 17 & $6(35,3 \%)$ & $3(17,6 \%)$ & $8(47,1 \%)$ \\
MAZO, J.Z. & 16 & $7(43,8 \%)$ & $8(50,0 \%)$ & $1(6,3 \%)$ \\
CAVICHIOLLI, F.R. & 14 & $1(7,1 \%)$ & $6(42,9 \%)$ & $7(50,0 \%)$ \\
SOARES, A.J.G. & 13 & $5(38,5 \%)$ & $4(30,8 \%)$ & $4(30,8 \%)$ \\
STIGGER, M.P. & 13 & $4(30,8 \%)$ & $4(30,8 \%)$ & $5(38,5 \%)$ \\
CAPRARO, A.M. & 12 & $2(16,7 \%)$ & $8(66,7 \%)$ & $2(16,7 \%)$ \\
VOTRE, S.J. & 11 & $5(45,5 \%)$ & $4(36,4 \%)$ & $2(18,2 \%)$ \\
VAZ, A.F. & 10 & $5(50,0 \%)$ & $4(40,0 \%)$ & $1(10,0 \%)$ \\
MEZZADRI, F.M. & 10 & $3(30,0 \%)$ & $4(40,0 \%)$ & $3(30,0 \%)$ \\
SILVA, P.P.C. & 10 & $4(40,0 \%)$ & $6(60,0 \%)$ & $0(0,0 \%)$ \\
FREITAS, C.M.S.M. & 9 & $4(44,4 \%)$ & $5(55,6 \%)$ & $0(0,0 \%)$ \\
LOVISOLO, H.R. & 9 & $6(66,7 \%)$ & $2(22,2 \%)$ & $1(11,1 \%)$ \\
REIS, H.H.B. & 8 & $2(25,0 \%)$ & $4(50,0 \%)$ & $2(25,0 \%)$ \\
\hline
\end{tabular}

Como se muestra en la tabla en cuestión, entre todos los investigadores que aparecen en el estudio realizado, Wanderley Marchi Júnior de la UFPR es el autor con el mayor número de artículos que moviliza la teoría elíasiana en el área. Es importante mencionar que Marchi Júnior realizó su doctorado bajo la orientación de Ademir Gebara entre finales de los años 1990 y principios de los años 2000 en la UNICAMP, donde muy probablemente pudo intensificar su contacto con la literatura de Norbert Elías en medio de las demandas de su tesis (Oliveira, 2018). Lo mismo también se puede decir sobre Souza y Starepravo, los cuales, al realizar sus formaciones stricto sensu en la UFPR, se acercaron más al referencial de Elías en virtud de disciplinas cursadas, participación en grupos de estudios y dinámicas de orientaciones. Estos son movimientos importantes y que sugieren un proceso de constitución de una generación intelectual que se inicia en la UNICAMP por vía del historiador Ademir Gebara y, en la secuencia, alcanza otras localidades de Brasil (Gebara, 2014; Oliveira, 2018).

Además del esfuerzo para reensamblar la génesis de esta familia intelectual es interesante también atenerse al modo con que tales agentes hacen uso de la teoría. Como pudimos localizar en los datos, los herederos de esa familia aparecen entre los investigadores que más operan a partir del "modo de trabajo" elíasiano, sugiriendo que, en alguna medida, el contacto de esa generación con el referencial representó avanzos para el proceso de apropiación general en el área. Hablando metafóricamente, donde los vientos del enfoque figuracional soplaron más fuerte, los agentes pudieron mejor sensibilizarse a esas contribuciones, a diferencia de otras localidades donde los usos de la teoría elíasiana son tímidos o desconocidos.

Dando continuidad a la exposición de los resultados, ahora se expone la cantidad de trabajos científicos por institución, así como el tipo de apropiación realizada en los artículos. 
Rev.int.med.cienc.act.fís.deporte - vol. 21 - número 82 - ISSN: 1577-0354

Figura 3. Número de artículos asociados a

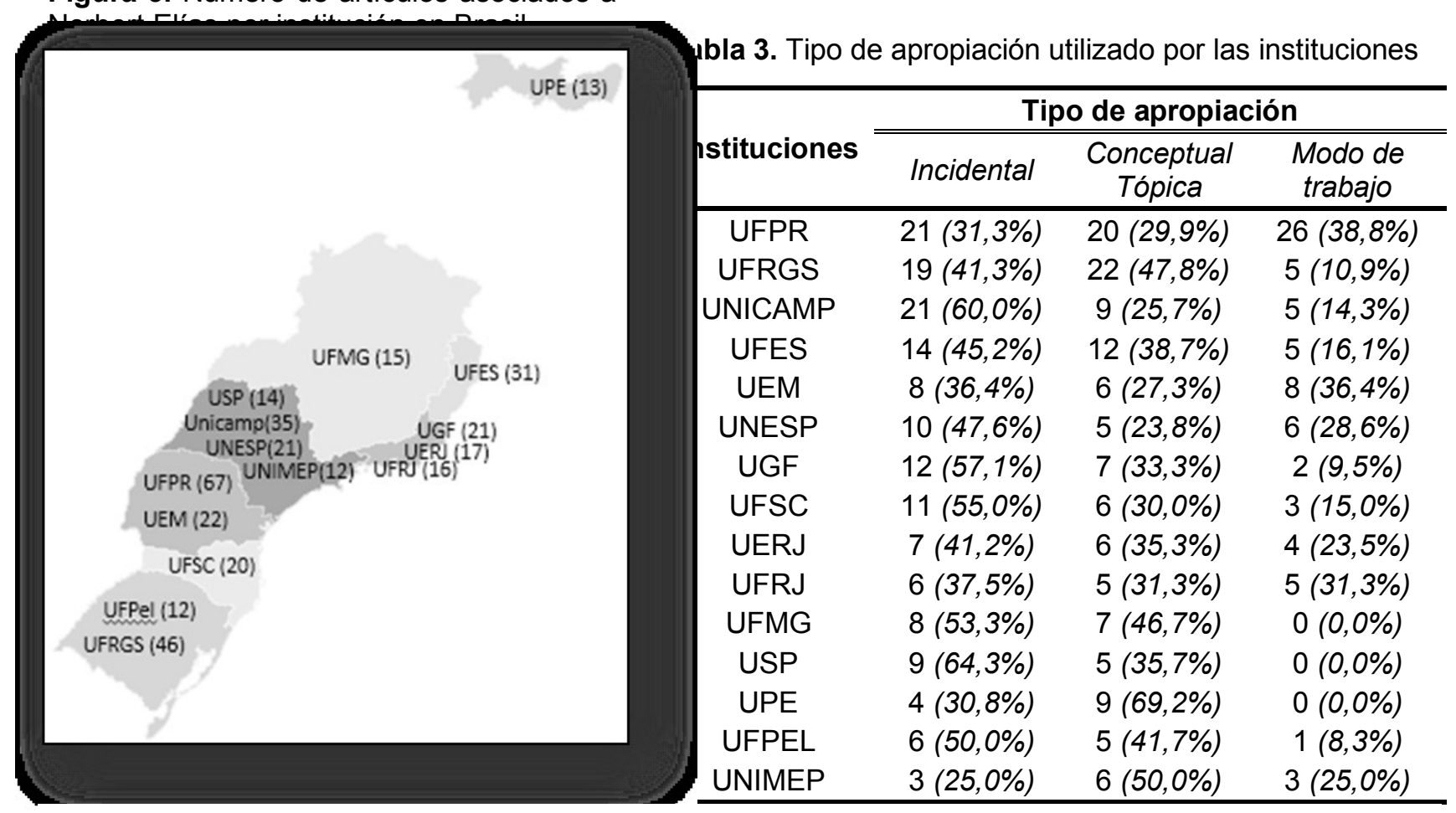

Nota: UFPR - Universidad Federal do Paraná; UFRGS - Universidad Federal do Rio Grande do Sul; UNICAMP - Universidad Estatal de Campinas; UFES - Universidad Federal do Espirito Santo; UEM Universidad Estatal de Maringá; UNESP - Universidad Estatal Paulista; UGF - Universidad Gama Filho; UFSC - Universidad Estatal de Santa Catarina; UERJ - Universidad Estatal do Rio de Janeiro; UFRJ Universidad Federal do Rio de Janeiro; UFMG - Universidad Federal de Minas Gerais; USP _

Universidad de São Paulo; UPE - Universidad de Pernambuco; UFPEL - Universidad Federal de Pelotas; UNIMEP - Universidad Metodista de Piracicaba

Como ya se vio en los resultados de la tabla 2, es posible notar en el mapa coroplético que la UFPR se trata de la institución que más posee artículos con mención al referencial teórico elíasiano en el área. Además, la universidad también presenta el mayor porcentaje de artículos producidos a partir de la apropiación del "modo de trabajo" del autor (tabla 3). Con respecto a las redes de colaboración, es interesante observar que la UEM exhibe un porcentaje similar para ese tipo de apropiación, lo que indica que existen trabajos confiables en el uso de los preceptos de la sociología figuracional de Elías. Estos resultados, a su vez, sugieren una transferencia de la herencia teórica elíasiana en el campo de la EF de una universidad a la otra, ampliando las redes de alcance de la teoría sociológica del autor en el área.

También se destaca en esta dinámica de relaciones establecida en el área de $\mathrm{EF}$, la UFRGS que ha operado sistemáticamente desde el marco de Elías, sin embargo, de una manera que podemos considerar más heterodoxo. Este uso identificado en la institución, indica que la teoría del sociólogo alemán no está en el centro de las discusiones de los investigadores, existiendo otros referenciales de soporte para el análisis de los objetos. Además, se infiere que la circulación del trabajo de Norbert Elías ejerció efectos de apropiación ortodoxa con menor intensidad en la UFGRS en comparación con la UFPR, lo 
que, en cierto modo, apunta a lógicas particulares de hacer ciencia social en EF.

En una dinámica diferente a la UFRGS, se encuentra la UNICAMP que no sólo tuvo una larga aproximación con el referencial teórico elíasiano a través del contacto directo con la red de sociólogos colaboradores de Elías, sino que también fue el epicentro en el proceso de ampliación de las redes de interdependencia académicas sobre la difusión del trabajo de Elías en el área. Por lo tanto, a través de un proceso de exportación de profesionales en el campo de la EF, la UNICAMP hizo distribuir su fuerza a otras regiones de Brasil (Oliveira, 2018).

En este contexto, a partir de la realidad basada en los datos, debe enfatizarse que los diferentes usos de la teoría de Elías en el campo de la EF brasileña apuntan a la conformación de una figuración de poder por el estatus de legitimidad en cuanto al manejo ortodoxo de la teoría, evidenciado en la existencia de grupos más preocupados por el referencial en cuestión y que se esfuerzan por hacer corresponder su modus operandi a los preceptos teóricos de esa familia intelectual. Dicho de otro modo, esa distribución desigual de la teoría, de alguna forma, reverbera en un desequilibrio interno de poder en el interior de la figuración (Elías, 2010).

En líneas generales, como se observó hasta el momento, el referencial teórico elíasiano se ha hecho eco en el campo académico, estando presente en varios estudios del área de EF. Sin embargo, el aumento en el número de proyectos en conexión con Elías no significa que el referencial ha sido manejado de forma sistemática. Por el contrario, lo que sucedió dentro de las regularidades fue un uso más heterodoxo o "incidental" de la teoría, caracterizado por una movilización más selectiva del referencial como se muestra a continuación. 
Rev.int.med.cienc.act.fís.deporte - vol. 21 - número 82 - ISSN: 1577-0354

Tabla 4: Obras / textos elíasianos más utilizados en las revistas científicas de EF

\begin{tabular}{lcc}
\hline Obras & $\mathbf{n}$ & $\boldsymbol{f}$ \\
\hline Deporte y ocio en el proceso de la civilización & 222 & $46,44 \%$ \\
El proceso de la civilización & 103 & $21,55 \%$ \\
Sociología fundamental & 36 & $7,53 \%$ \\
La sociedad de los Individuos & 29 & $6,07 \%$ \\
Los establecidos y los forasteros & 23 & $4,81 \%$ \\
La sociedad cortesana & 14 & $2,93 \%$ \\
Sobre el tiempo & 14 & $2,93 \%$ \\
Mozart: Sociología de un genio & 10 & $2,09 \%$ \\
Los alemanes & 8 & $1,67 \%$ \\
La soledad de los moribundos & 5 & $1,05 \%$ \\
Teoría del símbolo & 4 & $0,84 \%$ \\
Compromiso y distanciamiento & 2 & $0,42 \%$ \\
Escritos y ensayos & 2 & $0,42 \%$ \\
Sobre los seres humanos y sus emociones & 2 & $0,42 \%$ \\
Condición humana & 1 & $0,21 \%$ \\
Discurso sobre Adorno: Respeto y Crítica & 1 & $0,21 \%$ \\
Dynamics of sport groups with special reference to football & 1 & $0,21 \%$ \\
Norbert Elías por él mismo & 1 & $0,21 \%$ \\
\hline Total & $\mathbf{4 7 8}$ & $\mathbf{1 0 0 , 0 0 \%}$ \\
\hline Nota: *Sobre los seres humanos y sus emociones: un ensayo sobre la perspectiva
\end{tabular}

Después de determinar cuáles son los principales referenciales utilizados por los autores, se observó que la obra más citada entre los estudios observados es "Deporte y ocio en el proceso de la civilización", que aparece en casi el $50 \%$ de los artículos evaluados. En este contexto, como el deporte es uno de los temas más investigados en el área de EF, es probable que esa obra venga proporcionando importantes elementos teóricos para dimensionar sociológicamente el deporte. Sin embargo, la colección "Deporte y ocio en el proceso de la civilización" se trata, en rigor, de una obra que se alimenta de la teoría del proceso civilizador y, en ese sentido, puede y merece ser leída teniendo como base, por ejemplo, las discusiones que Elías emprendió en textos como el propio "El proceso de la civilización", "La sociedad cortesana" o "Los alemanes" (Elías y Dunning, 1992, Elías, 1993, 1997, 2001b, 2011). En cierto sentido, la obra magna de Elías, "El proceso de la civilización", ha recibido una atención más sistemática por parte de los autores que hacen uso de la teoría elíasiana en la literatura investigada. Lo mismo, al menos basado en la retomada cualitativa de los artículos aquí revisitados, no puede ser dicho para las otras obras mencionadas.

En este contexto, también es importante destacar que el libro "Sociología fundamental" como importante vía de acceso al pensamiento sociológico de Norbert Elías, ha sido poco citado en el campo de la EF en Brasil, al menos desde el punto de vista de la producción y socialización de artículos en las revistas científicas. Lo mismo se puede decir de obras como "La sociedad cortesana", "La sociedad de los individuos", y "Los alemanes" que, al lado de "El proceso de la civilización", constituyen el "núcleo-duro" de la teoría sociológica de Norbert Elías. Es evidente que esta condición puede presentar variaciones entre los investigadores y el hecho de que las mismas no sean 
citadas en los artículos no significa que no hayan sido leídas. En cualquier caso, esta discrepancia entre la movilización de los libros de Norbert Elías en las revistas de EF aquí investigadas, apunta a una dinámica que merece una mirada más cuidadosa por parte de quienes enseñan, desarrollan investigaciones y pretenden trabajar a partir de ese marco teórico.

En resumen, a través de los datos de la tabla 4, se observa que existe un gran potencial de la teoría de Elías a ser movilizada en el campo de los estudios socioculturales de EF, que por el momento fue poco explorado. Se llama la atención a obras como "La sociedad de los individuos" y "Teoría simbólica", en las cuales Elías hizo uso de su conocimiento en la esfera biológica para intentar defender el estudio global de los seres humanos, demostrando que la dicotomización entre las dimensiones biológicas y sociológicas es simplemente desprovista de realismo (Elías, 1994a, 1994b). Es decir, son, estrictamente hablando, contribuciones del acervo teórico elíasiano que aún no han sido debidamente explotadas y que podrían proporcionar bases epistemológicas para la construcción de una teoría de EF más realista y concordante a la transferencia progresiva de reflexividad en el área (Souza, 2019). Quizás un síntoma de este olvido es la propia distribución de las temáticas en que la teoría de Elías ha sido solicitada para orientar y basar la actividad científica del campo, como es posible visualizar en el organigrama a continuación.

Figura 4 - Principales temáticas vinculadas al referencial teórico elíasiano en las revistas científicas da EF

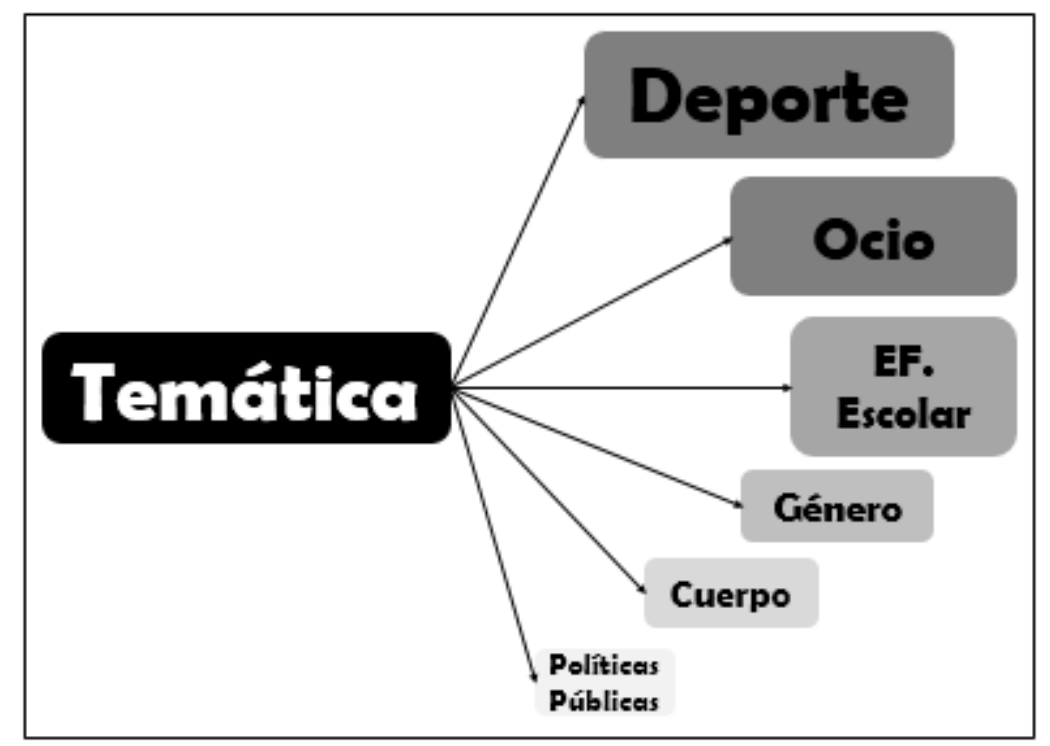

Al avanzar en el proceso de ordenar los documentos, fue posible verificar que el tema generativo predominante entre los textos en los que el referencial teórico elíasiano es movilizado, es el deporte, estando presente en aproximadamente el $60 \%$ de los manuscritos. En este escenario, se resaltan los estudios de la historia del deporte, prácticas de aventura, fútbol, luchas, mega eventos, medios, entre otros. Como segunda temática investigada aparecen los estudios del ocio, comprendiendo temas como turismo, ocio desviante, actividades en la naturaleza, historia del ocio, entre otros. En la secuencia, se encuentran investigaciones en el área de EF escolar, las cuales, 
han tenido un aumento en los últimos años. Entre las temáticas contempladas en ese segmento, se verifican las discusiones en torno a la violencia en las aulas, exclusión, infancia, gestión escolar, etc.

Es importante resaltar que, aunque no ha sido el objetivo aquí profundizar en cada uno de esos frentes donde la teoría elíasiana ha sido movilizada en el campo de la EF, debe tenerse en cuenta que las contribuciones de este autor han resonado significativamente en el área $e$ informado muchas investigaciones que buscan, de alguno modo, dialogar con el aporte teórico de la Sociología. Sin embargo, este panorama, aunque alentador, apunta a la supremacía de estudios en el campo del deporte y del ocio en detrimento de otras temáticas que también pueden y merecen ser investigadas por el prisma de la teoría sociológica de Elías. Ejemplo de ello, es la insípida cantidad de estudios acerca de la formación profesional en EF realizados a partir del referencial elíasiano. En ese sentido, se puede afirmar que la teoría de Elías ha sido dirigida más a las investigaciones satélites de la EF que propiamente al estudio ampliado del área para contribuir a su configuración epistemológica y profesional.

\section{CONSIDERACIONES FINALES}

Por medio de los datos analizados en esta investigación, es notable que la recepción del pensamiento sociológico elíasiano está en curso en el campo académico de EF. Como se señaló, los primeros estudios que hacen mención del trabajo de Elías en el interior de las revistas del área provienen de mediados de los años 1990. Teniendo en cuenta, por lo tanto, las discusiones acumuladas en lo artículo, se puede decir que el proceso de recepción de la teoría sociológica de Norbert Elías en el contexto brasileño es reciente y que el área de EF fue una puerta de entrada decisiva para la divulgación de su trabajo en el país (Oliveira, 2018).

En estos términos, aunque nos referimos a un proceso de recepción relativamente nuevo, está claro que éste se estableció de forma sistemática en el ámbito de EF a través de una familia intelectual elíasiana que inicialmente se formó como una pequeña configuración en el ámbito de la UNICAMP y luego se extendió a otras instituciones brasileñas, en especial para la UFPR que alberga un gran círculo de investigadores que operan a partir del marco teórico en cuestión. Cabe destacar, que la expansión de las configuraciones no se resume sólo al caso de esas dos instituciones, sino, por el contrario, el legado elíasiano también alcanzó otras regiones de Brasil, como se analiza a lo largo del texto.

Sin embargo, a pesar de esa expansión de las configuraciones de trabajo en torno del marco teórico elíasiano, eso no quiere decir que el rigor del proceso de apropiación corresponde a ese crecimiento. De hecho, al menos en el campo de la EF, los datos muestran que hay un uso mucho más heterodoxo de la teoría que una apropiación del "modo de trabajo" elíasiano. Esto quiere decir que la teoría sociológica del autor ha sido evocada de forma muy puntual, lo que, al principio, no indica problema alguno, excepto que una apropiación más sistemática en términos estructurales de campo aún está abierta, necesitando 
levar en cuenta aspectos centrales de la teoría, a ejemplo de las dinámicas a largo plazo poco observadas en los estudios, de la problematización de los cambios paralelos que se operan en la estructura de la sociedad y de la personalidad de los individuos, de las relaciones entre la distribución de la balanza de poder y el control de las emociones, sin mencionar la potencialidad de ese referencial para apoyar una teoría de la EF que desea superar la polarización "naturaleza" $X$ "cultura", tan rutinizada en el área.

A la manera de cierre, se resalta que, con respecto a la distribución de la teoría sociológica de Norbert Elías en el campo de la EF según las temáticas de investigación y los objetos investigativos elegidos, existe un predominio relativo de la movilización de ese referencial para llevar a cabo estudios sociológicos del deporte y del ocio. En este sentido, se entiende que lo más sofisticado de esta teoría aún está por ser apropiado y descubierto en el campo de la EF, a saber, la potencialidad de defenderse el movimiento humano como lenguaje (Elías, 1994b) y, sobre todo, la posibilidad de estructurarse así mismo una teoría sintética, no teleológica y realista de la EF (Elías, 2010) en que las dimensiones biológicas del movimiento no predominan sobre las dimensiones culturales del movimiento y viceversa, sino que constituyan un modelo integrativo (Elías, 1994a). De ahí la relevancia de estudios como el que se ha expuesto aquí, especialmente porque este tipo de diagnóstico permite repensar el estado de la teoría en el campo de la EF y, además, reflexionar si los investigadores promueven teorizaciones más o menos adherentes al área y que, de hecho, salvaguardan las especificidades de la profesión.

\section{REFERENCIAS BIBLIOGRÁFICAS}

Alfrey, L. y Gard, M. (2017). Figuring out the prevalence of fitness testing in physical education: A figurational analysis. European Physical Education Review, 25(1), 187-202, 2017. https://doi.org/10.1177/1356336X17715361

Betti, M. (1995). Um saber com sabor: "Da cultura do corpo" de Jocimar Daolio. Motriz, 1(2), 140-141.

Betti, M. (1996). A televisão e a Guerra do Pacaembu: "povão" versus "cidadãos". Revista Brasileira de Ciências do Esporte, 17(3), 220-225.

Black, J. y Fielding-Lloyd, B. (2017). Re-establishing the 'outsiders': English press coverage of the 2015 FIFA Women's World Cup. International Review for The Sociology of Sport, 54(3), 282-301. https://doi.org/10.1177/1012690217706192

Bourdieu, Pierre. (1968). "Campo intelectual e projeto criador". In J. Pouillon (Org.) Problemas do estruturalismo. Rio de Janeiro: Zahar.

Bourdieu, P. (1990). Coisas ditas. São Paulo: Brasiliense.

Brasil, M. R. (2018). Os usos da teoria sociológica de Pierre Bourdieu na área de Educação Física no Brasil (1977-2017). Dissertação de mestrado, Universidade Estadual de Maringá, Maringá, PR, Brasil.

Dunning, E. y Hughes, J. (2013). Norbert Elias and modern sociology: knowledge, interdependence, power, pocess. London: Bloomsbury.

Catani, A. M., Catani, D. B. y Pereira, G. R. M. (2001). As apropriações da obra de Pierre Bourdieu no campo educacional brasileiro, através de 
periódicos da área. Revista Brasileira de Educação, (17), 63-85. http://dx.doi.org/10.1590/S1413-24782001000200006

Elías, N. y Dunning, E (1992). Deporte y ocio en el proceso de la civilización. Madrid: Fondo de Cultura Económica.

Elías, N. (2010). Sociología fundamental. Barcelona: Gedisa.

Elias, N. (1993). O processo civilizador: formação do Estado e civilização. Rio de Janeiro: Jorge Zahar.

Elias, N. (1994a). A sociedade dos indivíduos. Rio de Janeiro: Jorge Zahar.

Elias, N. (1994b). Teoria simbólica. Oeiras: Celta.

Elias, N. (1997). Os alemães: A luta pelo poder e a evolução do habitus nos séculos XIX e XX. Rio de Janeiro: Jorge Zahar.

Elias, N. y Scotson J. L. (2000). Os Estabelecidos e os Outsiders: sociologia das relações de poder a partir de uma pequena comunidade. Rio de Janeiro: Jorge Zahar.

Elias, N. (2001a). Norbert Elias por ele mesmo. Rio de Janeiro: Jorge Zahar.

Elias, N. (2001b). A sociedade de corte. Rio de Janeiro: Jorge Zahar.

Elias, N. (2011). O processo civilizador: uma história dos costumes. 2. ed. Rio de Janeiro: Jorge Zahar.

Gebara, A. (2011). Simpósios Internacionais Processos Civilizadores [SIPC]: Apontamentos históricos. Educação e Fronteiras, 1(2), p. 147-53.

Gebara, A. Norbert Elias no Brasil. (2014). In A. Gebara, C. J. Costa y M. Sarat (Orgs) Leituras de Norbert Elias: processo civilizador, educação e fronteiras. Maringá: Eduem (pp. 21-34). https://doi.org/10.7476/9788576286523

Górnicka, B., Liston, K. y Mennell, S. (2015). Twenty-five years on: Norbert Elias's intellectual legacy 1990-2015. Human Figurations, 4(3).

Lavega-Burgués, P. y Navarro-Adelantado, V. (2019). Trazas del deporte en los juegos de Brueghel (S.XVI) y Rodrigo Caro (S.XVII). Revista Internacional de Medicina y Ciencias de La Actividad Física y del Deporte, 19(73),119-136. http://doi.org/10.15366/rimcafd2019.73.009

Manrique Arribas, J. C. (2018). La actividad físico-depotiva franquista como intento socializador de la juventud y la mujer. Revista Internacional de Medicina y Ciencias de La Actividad Física y del Deporte, 18(70), 303329. http://dx.doi.org/10.15366/rimcafd2018.70.007

Mennell, S. (2006). Civilizing processes. Theory, Culture \& Society, 2(2-3), 429431.https://doi.org/10.1177/026327640602300278

Morales-Rosillo, D. G., Petro, J. L. y Bonilla, D. A. (2019). Revisión integradora sobre proceso civilizatorio y violencia en boxeo y artes marciales mixtas. Ricyde. Revista Internacional de Ciencias del Deporte, 15(58), 413-432. https://doi.org/10.5232/ricyde2019.05808

Oliveira, V. M. (2018). A recepção do trabalho de Norbert Elias no Brasil: movimentos figuracionais a partir da área de Educação Física. Dissertação de mestrado, Universidade Estadual de Maringá, Maringá, PR, Brasil.

Pedraz, M. V. (2010). Educación Física e ideología. Creencias pedagógicas y dominación cultural en las enseñanzas escolares del cuerpo. Retos. Nuevas tendencias en Educación Física, Deporte y Recreación, (17), 7685. 
Resende, H. G. y Soares, A. J. G. (1996). Conhecimento e especificidade da Educação Física Escolar, na perspectiva da cultura corporal. Revista Paulista de Educação Física, 10(2), 49-59.

https://doi.org/10.11606/issn.2594-5904.rpef.1996.139648

Righeto, C. y Reis, H. H. B. (2017). Os árbitros de futebol e a mídia esportiva: a interpretação de árbitros paulistas sobre os comentários da mídia acerca do trabalho da equipe de arbitragem. Movimento, 23(1), 281-294. https://doi.org/10.22456/1982-8918.61652

Soares, A. J. G. (1995). "Jovens-velhos" esportistas eternamente? Movimento, 2(3), 17-26. https://doi.org/10.22456/1982-8918.2195

Souza J., Starepravo, F. A. y Marchi-Júnior, W. (2014). A sociologia configuracional de Norbert Elias - potencialidades e contribuições para 0 estudo do esporte. Revista Brasileira de Ciências do Esporte, 36(2), 429445. http://dx.doi.org/10.1590/S0101-32892014000200011

Souza, J. (2019). Educação Física Reflexiva - problemas, hipóteses e programa de pesquisa. Movimento, 25, 1-15.

https://doi.org/10.22456/1982-8918.78269

Número de citas totales / Total references: 33 (100\%)

Número de citas propias de la revista / Journal's own references: 2 $(6,06 \%)$

Rev.int.med.cienc.act.fís.deporte - vol. 21 - número 82 - ISSN: 1577-0354 